\title{
Bremsstrahlung and line spectroscopy of warm dense aluminum plasma heated by xuv free-electron-laser radiation
}

\author{
U. Zastrau, ${ }^{1, *}$ C. Fortmann, ${ }^{2}$ R. R. Fäustlin, ${ }^{3}$ L. F. Cao, ${ }^{1}$ T. Döppner, ${ }^{4}$ S. Düsterer, ${ }^{3}$ S. H. Glenzer, ${ }^{4}$ G. Gregori, ${ }^{5}$ \\ T. Laarmann, ${ }^{3}$ H. J. Lee, ${ }^{6}$ A. Przystawik, ${ }^{2}$ P. Radcliffe, ${ }^{3}$ H. Reinholz, ${ }^{2}$ G. Röpke, ${ }^{2}$ R. Thiele, ${ }^{2}$ J. Tiggesbäumker, ${ }^{2}$ \\ N. X. Truong, ${ }^{2}$ S. Toleikis, ${ }^{3}$ I. Uschmann, ${ }^{1}$ A. Wierling, ${ }^{2}$ T. Tschentscher, ${ }^{3}$ E. Förster, ${ }^{1}$ and R. Redmer ${ }^{2}$ \\ ${ }^{1}$ Institut für Optik und Quantenelektronik, Friedrich-Schiller-Universität, Max-Wien Platz 1, 07743 Jena, Germany \\ ${ }^{2}$ Institut für Physik, Universität Rostock, 18051 Rostock, Germany \\ ${ }^{3}$ Deutsches Elektronen-Synchrotron DESY, Notkestrasse 85, D-22607 Hamburg, Germany \\ ${ }^{4}$ Lawrence Livermore National Laboratory, University of California, P.O. Box 808, Livermore, California 94551, USA \\ ${ }^{5}$ Clarendon Laboratory, University of Oxford, Parks Road, Oxford OX1 3PU, United Kingdom \\ ${ }^{6}$ Department of Physics, University of California, Berkeley, California 94720, USA
}

(Received 25 April 2008; revised manuscript received 20 October 2008; published 30 December 2008)

\begin{abstract}
We report the creation of solid-density aluminum plasma using free-electron laser (FEL) radiation at $13.5 \mathrm{~nm}$ wavelength. Ultrashort pulses were focused on a bulk Al target, yielding an intensity of 2 $\times 10^{14} \mathrm{~W} / \mathrm{cm}^{2}$. The radiation emitted from the plasma was measured using an xuv spectrometer. Bremsstrahlung and line intensity ratios yield consistent electron temperatures of about $38 \mathrm{eV}$, supported by radiation hydrodynamics simulations. This shows that xuv FELs heat up plasmas volumetrically and homogeneously at warm-dense-matter conditions, which are accurately characterized by xuv spectroscopy.
\end{abstract}

DOI: 10.1103/PhysRevE.78.066406

\section{INTRODUCTION}

The physics of warm dense matter (WDM) [1] has gained increasing interest because of its location in the transition region from cold condensed materials to hot dense plasmas. WDM states are of paramount importance to model astrophysical objects such as giant planets [2] or brown dwarfs [3]. Furthermore, WDM occurs as a transient state in novel experiments to generate high-energy densities in materials, most notably the realization of inertial confinement fusion $[4,5]$. The first experimental investigations of WDM have been performed, e.g., with shock-wave experiments [6] and with laser-excited plasmas [7-11].

WDM describes materials at temperatures of several $\mathrm{eV}$ at solidlike densities. Its creation and investigation under controlled conditions in the laboratory is a difficult task. Using common optical short-pulse lasers, nonlinear absorption leads to rapid temporal variations, steep spatial gradients, and a broad spectrum of plasma physical processes. Pioneering techniques such as laser-driven shock heating, x-ray heating, ion-heating techniques $[6,12-15]$, and $x$-ray Thomson scattering [16] have been developed in order to improve the plasma heating mechanism.

In WDM the electron temperature is comparable to the Fermi energy, i.e., the degeneracy parameter $\theta=k_{\mathrm{B}} T_{\mathrm{e}} / E_{\mathrm{F}}$ is close to unity. Furthermore, the ion coupling parameter $\Gamma$ $=Z^{2} / 4 \pi \epsilon_{0} k_{\mathrm{B}} T_{\mathrm{i}}\left(4 \pi n_{\mathrm{i}} / 3\right)^{1 / 3}$ is greater than or equal to unity, i.e., the interparticle Coulomb correlation energy is equal or exceeds the thermal energy; $Z$ is the ion charge, and $n_{\mathrm{i}}$ is the ion density. Thus, electrons as well as ions exhibit strong temporal and spatial correlations which depend strongly on the plasma parameters, temperature, and density.

A proper description of WDM is also a tremendous challenge to many-particle physics. Both the theory for ideal

\footnotetext{
*zastrau@ioq.uni-jena.de
}

PACS number(s): 52.50.Jm, 52.25.Os, 52.27.Gr, 52.70.La plasmas and for condensed matter fail in this regime. Classical plasma theory based on expansions of the correlation contributions in powers of the coupling parameter breaks down since $\Gamma \geqslant 1$, and strong-coupling effects among the various species have to be taken into account. On the other hand, the plasma is too hot to be considered as condensed matter, i.e., expansions in terms of the degeneracy parameter $\theta$ also fail. Thus, precise knowledge of physical properties as a function of the plasma parameters temperature and density is of primary importance.

In this article, we demonstrate that xuv free-electron lasers (FELs) open a promising possibility to heat matter volumetrically and homogeneously. Furthermore, we show that xuv bremsstrahlung and line spectroscopy allow the determination of the plasma temperature and free-electron density.

\section{EXPERIMENTAL SETUP}

\section{A. Free-electron laser characteristics}

The fourth-generation light source FLASH (free-electron laser in Hamburg) uses the self-amplified spontaneous emission (SASE) principle to generate brilliant xuv pulses $[17,18]$. In the experiment, pulses of $91.8 \mathrm{eV}$ photon energy (wavelength $\lambda=13.5 \mathrm{~nm}),(15 \pm 5)$ fs duration were focused to a $30-\mu \mathrm{m}$ spot by a carbon-coated ellipsoidal mirror with grazing incidence angle of $3^{\circ}$ and $2 \mathrm{~m}$ focal length, as shown in Fig. 1.

Since the FEL process starts from spontaneous radiation, it shows an intrinsic shot-to-shot pulse energy spread. The relevant distribution of pulse energies for this experiment is shown in Fig. 2. The total number of pulses in our measurement was 81000 , including $3 \%$ of zero-energy events and a significant fraction of high-energy pulses up to $130 \mu \mathrm{J}$. The mean value is $48 \mu \mathrm{J}$, measured at the end of the undulators. Since the beamline transmission is known to be $T=0.68$, the average pulse energy at the experiment is $33 \mu \mathrm{J}$. 

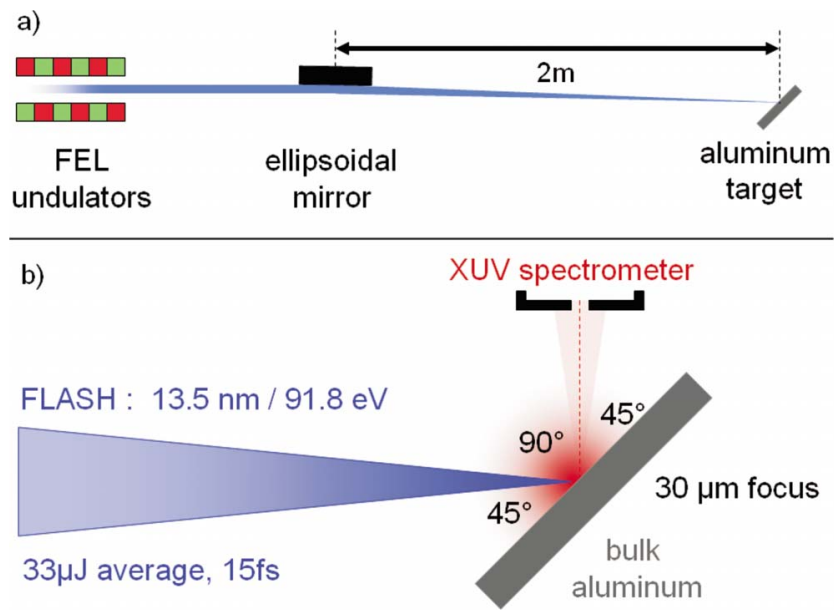

FIG. 1. (Color online) Experimental setup. (a) The xuv pulses from the FEL undulators are focused by an elliptical beamline mirror on the bulk aluminum target, creating a $30-\mu \mathrm{m}$ focus. (b) The target is hit under $45^{\circ}$ and plasma is created. A high-resolution xuv spectrometer observes the plasma emission under $45^{\circ}$ to the surface normal.

\section{B. xuv plasma spectroscopy}

Each pulse irradiates a bulk aluminum target under $45^{\circ}$ incident angle, resulting in an average intensity of 2 $\times 10^{14} \mathrm{~W} / \mathrm{cm}^{2}$; see Fig. 1 . The polarization is linear in the horizontal plane. At the chosen wavelength, the critical density for penetration into the bulk, $n_{\text {crit }}=(2 \pi c)^{2} \epsilon_{0} m_{\mathrm{e}} / e^{2} \lambda^{2}$ $=6.1 \times 10^{24} \mathrm{~cm}^{-3}$, is about 40 times higher than the valence electron density in cold solid aluminum, $n_{\mathrm{e}}=1.6$ $\times 10^{23} \mathrm{~cm}^{-3}$; therefore, the initial absorption length is $40 \mathrm{~nm}$ [19]. The pulse energy is deposited in a target volume of $\pi$ $\times(15 \mu \mathrm{m})^{2} \times 40 \mathrm{~nm}$, generating WDM. The number of $10^{12}$ atoms in this volume is in the same order of magnitude as the incident photon number.

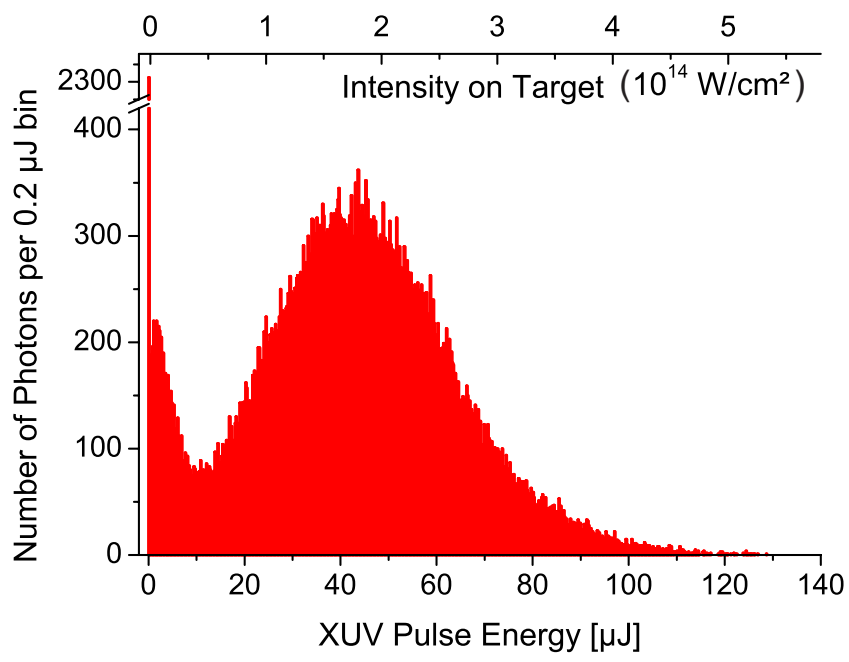

FIG. 2. (Color online) Histogram of the energy spread of 13.5-nm xuv pulses generated by self-amplified spontaneous emission (SASE). Only pulses contributing to the measured spectrum of the performed experiment are shown. The top ordinate shows the corresponding irradiation intensities on target.

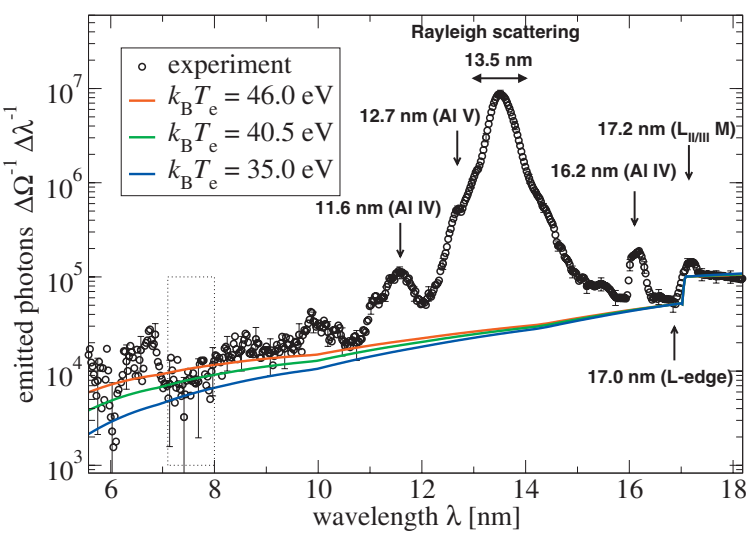

FIG. 3. (Color) Experimental xuv photon spectrum per solid angle $\Delta \Omega=4 \times 10^{-4} \mathrm{sr}$ and wavelength interval $\Delta \lambda=0.025 \mathrm{~nm}$ (symbols with error bars) and bremsstrahlung calculations for different electron temperatures. The spectrum is corrected for the spectrometer throughput and detection efficiency.

At fixed target position, the emission spectrum of about $10^{4}$ exposures was recorded before moving to a fresh site. Since the very first FEL pulse ablates a few-nanometer-thin surface layer, a further surface cleaning technique was not necessary. The target was at ambient temperature, and vacuum was kept constant at $10^{-7}$ mbar.

The FEL was run in multibunch operation mode at a $5-\mathrm{Hz}$ repetition rate, with 20 bunches per train giving $100 \mathrm{FEL}$ pulses per second. Five separate measurements of different durations adding up to a total interaction time of $13.5 \mathrm{~min}$ were performed. Since the individual spectra look identical, we assume that plasma formation and emission processes vary very slowly during the measurement.

xuv emission spectra in the region of 6-18 nm were measured with a high-throughput spectrometer, featuring a toroidal Ni-coated focusing mirror and a free-standing transmission grating. The spectrometer is described in detail in Ref. [20]. The spectral resolution was limited to $0.2 \mathrm{~nm}$ due to a slightly fluctuating plasma position. A back-thinned xuv charge-coupled device (CCD) camera with $13 \times 13 \mu \mathrm{m}^{2}$ pixel size and a quantum efficiency of $\eta=0.45$ served as the detector. From the measured spectra, absolute photon numbers per wavelength interval and solid angle are calculated using the tabulated efficiency of all components.

\section{DATA ANALYSIS}

Figure 3 shows the sum of all spectra in logarithmic scale after correction for the spectrometer throughput and detection efficiency. The error bars arise essentially from statistical signal-to-noise ratios beside uncertainties of the spectrometer components. The main peak at $13.5 \mathrm{~nm}$ stems from Rayleigh scattering of FEL photons by bound aluminum electrons. It is broadened symmetrically by $\pm 0.4 \mathrm{~nm}$ due to artifacts originating from the support grid of the transmission grating. Spectral lines from Al IV and Al V are identified using the NIST tables [21] as listed in Table I. The continuum emission is formed by free-free transition radiation (bremsstrahlung) and free-bound recombination radiation. 
TABLE I. List of the spectral lines emitted from the plasma, identified with the NIST database [21,23].

\begin{tabular}{|c|c|c|c|c|}
\hline $\begin{array}{l}\text { Experimental } \\
\text { data } \\
(\mathrm{nm})\end{array}$ & $\begin{array}{c}\text { Reference } \\
\text { data } \\
(\mathrm{nm})\end{array}$ & $\begin{array}{c}\text { Relative } \\
\text { intensity } \\
\text { (NIST) }\end{array}$ & $\begin{array}{l}\text { Oscillator } \\
\text { strength } f \\
\text { (NIST) }\end{array}$ & Transition \\
\hline $11.6 \pm 0.2$ & 11.646 & 250 & 0.332 & $\begin{array}{c}\text { Al IV: } 2 s^{2} 2 p^{6} \\
-2 s^{2} 2 p^{5}\left({ }^{2} p_{1 / 2}^{0}\right) 4 d\end{array}$ \\
\hline $12.7 \pm 0.2$ & 12.607 & 800 & & $\begin{array}{l}\text { Al V: } 2 s^{2} 2 p^{5} \\
-2 s^{2} 2 p^{4}\left({ }^{1} D\right) 3 s\end{array}$ \\
\hline $16.2 \pm 0.2$ & 16.169 & 700 & 0.247 & $\begin{array}{c}\text { Al IV: } 2 s^{2} 2 p^{6} \\
-2 s^{2} 2 p^{5}\left({ }^{2} p_{1 / 2}^{0}\right) 3 s\end{array}$ \\
\hline $17.2 \pm 0.2$ & 17.14 & & & $L_{\mathrm{II} / \mathrm{III}} M$ \\
\hline
\end{tabular}

Since the target is heated volumetrically, the continuum emission is partly reabsorbed and we observe a steplike feature at $17.0 \mathrm{~nm}$ that is consistent with the absorption $L_{\mathrm{II} / \mathrm{III}}$ edge [19]. This indicates deep deposition of energy into the target, as expected for xuv photon-matter interactions. The corresponding absorption $L$ edge at a similar excitation flux was also analyzed in laser-excited silicon [22]. Finally, the $L_{\mathrm{II} / \mathrm{III}} M$ fluorescence line [23] is observed at $17.2 \mathrm{~nm}$.

\section{A. Bremsstrahlung}

The experimental spectra allow the determination of the plasma parameters using fundamental relations [24]. The electron temperature and density are inferred from the continuum background radiation due to bremsstrahlung. We compare the experimental data to Kramers' law [25]

$$
j_{\mathrm{ff}}(\lambda)=\left(\frac{e^{2}}{4 \pi \epsilon_{0}}\right)^{3} n_{\mathrm{e}}^{2} \frac{16 \pi Z e^{-2 \pi \hbar c / \lambda k_{\mathrm{B}} T_{\mathrm{e}}}}{3 m_{\mathrm{e}} c^{2} \lambda^{2} \sqrt{6 \pi k_{\mathrm{B}} T_{\mathrm{e}} m_{\mathrm{e}}}} g_{T}(\lambda)
$$

for the free-free emissivity $j_{\mathrm{ff}}(\lambda)$. Here, $m_{\mathrm{e}}$ is the electron mass and $g_{\mathrm{T}}(\lambda)$ is the wavelength-dependent Gaunt factor [26], accounting for medium and quantum effects. It is calculated in a Sommerfeld approximation [27]. We assume an average ion charge of $Z=4$, which is supported by calculations of the relative ion abundances using the code COMPTRA04 [28]; see below. In the wavelength range from $7.1 \mathrm{~nm}$ to $8.0 \mathrm{~nm}$ (marked by the dotted box in Fig. 3), we expect no essential contributions from bound-bound and bound-free transitions. Statistical analysis of the data in this range yields $40.5 \mathrm{eV}$ for the temperature with a rms error of $\pm 5.5 \mathrm{eV}$. Bremsstrahlung spectra for $35 \mathrm{eV}, 40.5 \mathrm{eV}$, and $46 \mathrm{eV}$ are shown in Fig. 3. Reabsorption was considered using tabulated opacity data [19]; in this way, the $L$ edge at $17.0 \mathrm{~nm}$ is reproduced. The height of the $L$ edge corresponds to a transmission through $40 \mathrm{~nm}$ of cold aluminum.

Kramers' law [Eq. (1)] depends on the square of $n_{e}$. From the absolute photon number at $\lambda=17.0 \mathrm{~nm}, N_{\text {photon }}$ $=51424 \Delta \Omega^{-1} \Delta \lambda^{-1}$, we calculate the free-electron density using Eq. (1) as $n_{\mathrm{e}}=4.0 \times 10^{22} \mathrm{~cm}^{-3}$, taking the inferred plasma temperature of $40.5 \mathrm{eV}$. This value is consistent with radiation hydrodynamics simulations; see below.

\section{B. Transition line ratio}

Independently, the electron temperature can be obtained from the ratio of integrated line intensities $I_{\nu}$ for the identified transition lines from the Boltzmann distribution [29] as follows:

$$
\frac{I_{1}}{I_{2}}=\frac{\omega_{1}^{3}}{\omega_{2}^{3}} \frac{f_{1}}{f_{2}} e^{-\hbar\left(\omega_{1}-\omega_{2}\right) / k_{\mathrm{B}} T_{\mathrm{e}}},
$$

with the corresponding photon frequencies $\omega_{\nu}$ and oscillator strengths $f_{\nu}$, having in mind that the plasma is optically thin. Here, the Al IV lines (doublets) at $\lambda_{1}=16.169 \mathrm{~nm}$ and $\lambda_{2}$ $=11.646 \mathrm{~nm}$, with oscillator strengths given in Table I, are used. Integration was performed from 15.9 to $16.3 \mathrm{~nm}$ and from 10.8 to $11.9 \mathrm{~nm}$ after subtraction of the bremsstrahlung continuum, respectively. The resulting temperature is $(34 \pm 6) \mathrm{eV}$. Within the error bounds, this is consistent with the temperature inferred by analysis of the bremsstrahlung continuum, so that we can state the plasma temperature with about $38 \mathrm{eV}$.

A full compliance of line and continuum temperature cannot be expected, since the plasma dynamics affects both emission processes in different ways. At early times after the laser-target interaction, the system is still very dense and the excited levels of the transitions under consideration are possibly dissolved into the continuum. Only after expansion are the excited levels well defined and radiative transitions take place. Bremsstrahlung, on the other hand, is most relevant at early times due to the $n_{\mathrm{e}}^{2}$ dependence of its emissivity; see Eq. (1). Thus, in the bremsstrahlung emission we expect a higher temperature than in the line spectrum.

\section{Relative ion abundance}

The relative abundance of aluminum ions was calculated with the code COMPTRA04 [28]. Results for electron temperatures from 10 to $50 \mathrm{eV}$ are shown in Fig. 4, assuming a solid density $\rho_{\text {sol }}=2.7 \mathrm{~g} / \mathrm{cm}^{3}$ and $0.5 \rho_{\text {sol }}$-i.e., slightly expanded. The concentration of ion species for the relevant temperatures between 34 and $40 \mathrm{eV}$ complies with the observed line emission spectrum. At an averaged temperature of $38 \mathrm{eV}$, the ion fractions of $\mathrm{Al} \mathrm{IV}, \mathrm{Al} \mathrm{V}$, and $\mathrm{Al}$ VI take the values of about $7 \%, 45 \%$, and $46 \%$, respectively. Amounts of Al I-III as well as Al VII and higher are negligible.

All expected spectral lines from Al IV in the observed spectral range are either observed (at $16.2 \mathrm{~nm}$ ) or covered by the strong FEL signal (at $12.9 \mathrm{~nm}$ ). For transition details see Table I. Weak lines from Al v between 11.9 and $13.2 \mathrm{~nm}$ overlap with the Rayleigh peak and only the 12.607-nm line can be identified. Transition lines from Al VI, which are located between 8.6 and $10.9 \mathrm{~nm}$, are not significantly present compared to the detector noise. This indicates that the corresponding high-lying excited levels are dissolved and do not contribute.

Emission lines from Al VII (and higher) are expected to play a significant role only at temperatures exceeding $40 \mathrm{eV}$, as shown in Fig. 4. These lines have also not been found, but have previously been observed in optical laser-matter interaction experiments [30]. This contrast is well understood by 


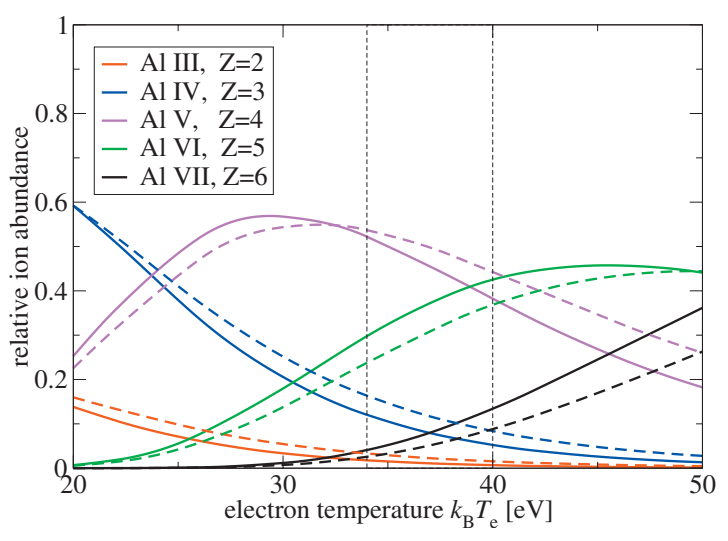

FIG. 4. (Color) Calculation of the relative $\mathrm{Al}$ ion species abundance from COMPTRA04 as a function of the electron temperature. The solid line is for the $\mathrm{Al}$ density $\rho_{\mathrm{sol}}=2.7 \mathrm{~g} / \mathrm{cm}^{3}$, dashed line $0.5 \rho_{\mathrm{sol}}=1.35 \mathrm{~g} / \mathrm{cm}^{3}$.

scrutinizing the different mechanisms of absorption and ionization in the case of optical light as opposed to the case of xuv photons.

\section{DISCUSSION}

\section{A. Different photon absorption mechanisms}

In optical laser-matter interactions, dominant absorption mechanisms are multiphoton ionization, nonlinear processes, inverse bremsstrahlung, and resonance absorption [31]. When the critical free-electron density of the optical laser is exceeded, most light is reflected and absorption is limited to the skin layer, leaving behind a plasma with steep density and temperature gradients.

For xuv photons, nonlinear absorption is negligible at the considered intensity and resonance absorption is not important since the plasma is undercritical. Thus, "hot"-electron production in the $\mathrm{keV}-\mathrm{MeV}$ range is unlikely. Photoexcitation and inverse bremsstrahlung are the only possible mechanisms. Since there are no prepulses in the FEL case, the deposition of photons starts in a cold target and the energy is distributed volumetrically and homogeneously throughout the interaction zone.

A change in the polarization of the FEL (e.g., using circularly polarized light) could lead to less heating and certainly to a reduction in the scattered intensity (Rayleigh peak). For atomic systems, a systematic decrease in the photoionization yield has been reported upon changing the laser's polarization [32], while such studies for solid-state targets at xuv conditions remain to be done.

The plasma production mechanism presented here takes advantage of photons exceeding a $2 p$-level binding energy of $72.8 \mathrm{eV}$. In particular, a $2 p$ bound electron is photoionized with a cross section of $\sigma_{\mathrm{PI}}=7 \mathrm{Mbarn}$ [19]. This electron is transferred into the conduction band, leaving behind a hole in the $2 p$ shell. For partially ionized aluminum the photoabsorption cross section increases below the $L_{\mathrm{II} / I I I}$ edge slightly with temperature [33]. Due to their high excess energy of about $20 \mathrm{eV}$, further electrons at lower energies are excited via impact ionization and Auger processes [1]. The electrons (a) Electron density $\left[1 / \mathrm{cm}^{3}\right]$

(b) Electron temperature

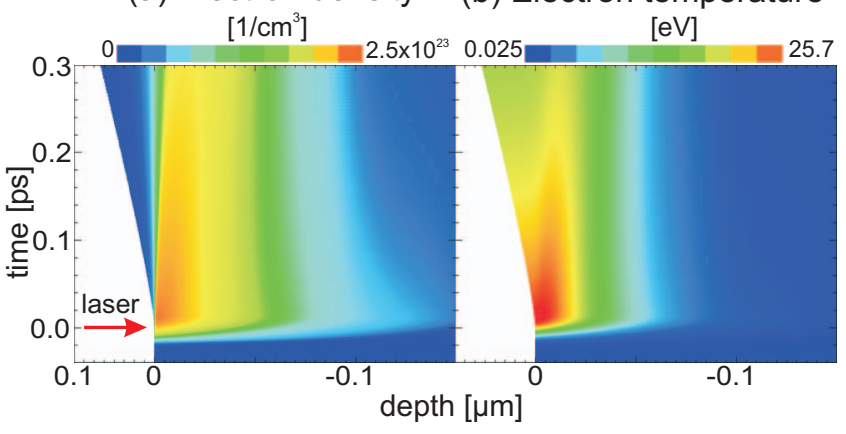

FIG. 5. (Color) HELIOS simulation results for the electron density (left) and electron temperature (right) as a function of time and radius.

equilibrate at a temperature of several $\mathrm{eV}$ up to hundred femtoseconds $[34,35]$, forming typical WDM. During this time, electrons can also recombine with the $2 p$ holes by emitting fluorescence radiation at $17.2 \mathrm{~nm}$ wavelength. This state of matter cools down on a picosecond time scale by energy transfer to the lattice via electron-phonon scattering [24].

\section{B. Hydrodynamics simulation}

To illustrate the hydrodynamic processes and to estimate the electron temperature, one-dimensional radiation hydrodynamics simulations using HELIOS [36] have been performed. HELIOS features a Lagrangian reference framei.e., the grid moves with fluid, separate ion and electron temperatures, and flux-limited Spitzer thermal conductivity. It allows the deposition of laser energy via inverse bremsstrahlung as well as bound-bound and bound-free transitions, using a SESAME-like equation of state. Per atom, 2.6 conduction band electrons were assumed to contribute to the laser absorption [37]. The results are shown in Fig. 5. On the time scale of the FEL pulse, both electron density and temperature rise up to values of $n_{\mathrm{e}} \simeq 10^{23} \mathrm{~cm}^{-3}$ and $k_{\mathrm{B}} T_{\mathrm{e}}$ $\simeq 26 \mathrm{eV}$, respectively, without any steep gradient. These values are in good qualitative agreement with the results for $n_{\mathrm{e}}$ and $T_{\mathrm{e}}$ obtained by the spectral analysis.

This hydrodynamics simulation uses an average pulse energy of $33 \mu \mathrm{J}$ on the target as an input parameter. As discussed above, Fig. 2 illustrates that a significant fraction of pulses have much higher pulse energies up to $130 \mu \mathrm{J}$. Since the free-free emissivity [Eq. (1)] depends strongly nonlinearly on both electron temperature and density, we expect the observed radiation to be rather dominated by the high-energy fraction of the xuv pulses than by its average value. This finally explains the slight underestimation of the electron temperature in the hydrodynamics simulation compared to the experimental results.

Additionally, the simulation shows that these WDM conditions exist for about $200 \mathrm{fs}$, at almost constant plasma density and temperature, and hydrodynamic motion is negligible [38]. Plasma expansion as well as electron diffusion to the cold matter of the bulk target and heat conduction becomes important during the first several picoseconds [39], while the density decreases about a factor of 2 , influencing the relative 
abundance of ion species only slightly, as demonstrated in Fig. 4.

\section{SUMMARY AND CONCLUDING REMARKS}

The capability of xuv FEL radiation to create WDM by interaction with a solid aluminum target was demonstrated. The analysis of the xuv line and continuum emission spectra yields an electron temperature of $(34 \pm 6) \mathrm{eV}$ and $(40.5 \pm 5.5) \mathrm{eV}$, respectively. The observed line spectrum is compatible with predicted ion abundances. Together with radiation hydrodynamics modeling, we get a sound picture of complex xuv laser-plasma interaction dynamics. The simulations confirm the volumetric heating of the target without strong gradients. Our results provide complementary information to results that were reported for optical laser-matter interactions [30].

Further and detailed studies of WDM will include spatially and temporally resolved experiments to determine the electron temperature and density. For this regime, novel diagnostic techniques such as X-ray interferometry [40,41] and x-ray Thomson scattering $[34,42,43]$ have been developed. In combination with these techniques, the xuv FEL will be a unique platform for WDM investigations. This will be im- portant for shock-wave physics, applied-material studies, planetary physics and inertial confinement fusion, and other forms of high-energy density-matter generation.

\section{ACKNOWLEDGMENTS}

We thankfully acknowledge financial support by the German Helmholtzgemeinschaft via the Virtual Institute VH-VI104, the German Federal Ministry for Education and Research via Project No. FSP 301-FLASH, and the Deutsche Forschungsgemeinschaft (DFG) via the Sonderforschungsbereich SFB 652. T.L. acknowledges financial support from the DFG under Grant No. LA 1431/2-1. R.R.F. received DFG funds via Grant No. GRK 1355. The work of S.H.G. was performed under the auspices of the U.S. Department of Energy by Lawrence Livermore National Laboratory under Contract No. DE-AC52-07NA27344. S.H.G. was also supported by LDRDs 08-ERI-002, 08-LW-004, and the Alexander von Humboldt foundation. The work of G.G. was partially supported by the Science and Technology Facilities Council of the United Kingdom. Finally, the authors are greatly indebted to the machine operators, run coordinators, and scientific and technical teams of the FLASH facility for enabling an outstanding performance.
[1] R. W. Lee et al., Laser Part. Beams 20, 527 (2002).

[2] N. Nettelmann et al., Astrophys. J. 683, 1217 (2008).

[3] D. Saumon et al., High Press. Res. 16, 331 (2000).

[4] J. D. Lindl et al., Phys. Plasmas 11, 339 (2004).

[5] T. R. Dittrich et al., Phys. Plasmas 6, 2164 (1999).

[6] L. B. Da Silva et al., Phys. Rev. Lett. 78, 483 (1997).

[7] Y. Ping, D. Hanson, I. Koslow, T. Ogitsu, D. Prendergast, E. Schwegler, G. Collins, and A. Ng, Phys. Rev. Lett. 96, 255003 (2006).

[8] T. Ao, Y. Ping, K. Widmann, D. F. Price, E. Lee, H. Tam, P. T. Springer, and A. Ng, Phys. Rev. Lett. 96, 055001 (2006).

[9] K. Widmann, T. Ao, M. E. Foord, D. F. Price, A. D. Ellis, P. T. Springer, and A. Ng, Phys. Rev. Lett. 92, 125002 (2004).

[10] G. W. Collins, P. M. Celliers, L. B. Da Silva, R. Cauble, D. M. Gold, M. E. Foord, N. C. Holmes, B. A. Hammel, R. J. Wallace, and A. Ng, Phys. Rev. Lett. 87, 165504 (2001).

[11] A. Saemann, K. Eidmann, I. E. Golovkin, R. C. Mancini, E. Andersson, E. Forster, and K. Witte, Phys. Rev. Lett. 82, 4843 (1999).

[12] T. S. Perry et al., Phys. Rev. E 54, 5617 (1996).

[13] J. J. MacFarlane et al., Phys. Rev. E 66, 046416 (2002).

[14] P. K. Patel, A. J. Mackinnon, M. H. Key, T. E. Cowan, M. E. Foord, M. Allen, D. F. Price, H. Ruhl, P. T. Springer, and R. Stephens, Phys. Rev. Lett. 91, 125004 (2003).

[15] S. Sakabe, R. Sigel, G. D. Tsakiris, I. B. Foldes, and P. Herrmann, Phys. Rev. A 38, 5756 (1988).

[16] S. H. Glenzer, G. Gregori, R. W. Lee, F. J. Rogers, S. W. Pollaine, and O. L. Landen, Phys. Rev. Lett. 90, 175002 (2003).

[17] W. Ackermann et al., Nat. Photonics 1, 336 (2007).

[18] V. Ayvazyan et al., Phys. Rev. Lett. 88, 104802 (2002).

[19] B. L. Henke et al., At. Data Nucl. Data Tables 54, 181 (1993).
[20] J. Jasny et al., Rev. Sci. Instrum. 65, 1631 (1994).

[21] V. Kaufman et al., J. Phys. Chem. Ref. Data 20, 775 (1991).

[22] K. Oguri et al., Appl. Phys. Lett. 87, 011503 (2005).

[23] J. A. Bearden, Rev. Mod. Phys. 39, 78 (1967).

[24] H. R. Griem, Principles of Plasma Spectroscopy (Cambridge University Press, Cambridge, England, 1997).

[25] H. A. Kramers, Philos. Mag. 46, 836 (1923).

[26] J. A. Gaunt, Proc. R. Soc. London, Ser. A 126, 654 (1930).

[27] C. Fortmann et al., High Energy Density Phys. 2, 57 (2006).

[28] S. Kuhlbrodt et al., Contrib. Plasma Phys. 45, 73 (2005).

[29] W. Lochte-Holtgreven, in Plasma Diagnostics, edited by W. Lochte-Holtgreven (AIP, New York, 1995), p. 135.

[30] U. Teubner et al., Appl. Phys. Lett. 59, 2672 (1991).

[31] P. Gibbon et al., Plasma Phys. Controlled Fusion 38, 769 (1996).

[32] K. J. LaGattuta, Phys. Rev. A 43, 5157 (1991).

[33] M. Fajardo et al., Eur. Phys. J. D 29, 69 (2004).

[34] T. Tschentscher et al., Eur. Phys. J. D 36, 193 (2005).

[35] A. L. Dobryakov et al., Phys. Scr. 60, 572 (1999).

[36] J. MacFarlane et al., J. Quant. Spectrosc. Radiat. Transf. 99, 381 (2006).

[37] R. LaVilla et al., Phys. Rev. Lett. 9, 149 (1962).

[38] M. M. Murnane et al., Science 251, 531 (1991).

[39] A. Krenz et al., Eur. Phys. J. D 36, 199 (2005).

[40] L. B. Da Silva, T. W. Barbee, R. Cauble, P. Celliers, D. Ciarlo, S Libby, R. A. London, D. Matthews, S. Mrowka, J. C. Moreno, D. Ress, J. E. Trebes, A. S. Wan, and F. Weber, Phys. Rev. Lett. 74, 3991 (1995).

[41] J. Filevich et al., Appl. Opt. 43, 3938 (2004).

[42] S. H. Glenzer et al., Phys. Rev. Lett. 98, 065002 (2007).

[43] A. Höll et al., High Energy Density Phys. 3, 120 (2007). 